nahezukommen. Dieses Bemühen hat seine Ausprägung im „self-restraint“ gefunden. Die Verfassungsgerichte lehnen es grundsätzlich $a b$, in das gesetzgeberische Ermessen einzugreifen.

Während in Deutschland wegen der detailliert und präzise gefaßten Verfassung das Problem der Zuordnung zur politischen oder rechtlichen Sphäre seltener auftritt, befindet sich der Supreme Court der Vereinigten Staaten von Amerika sehr viel häufiger in diesem Dilemma, weil die amerikanische Verfassung wesentlich mehr dem freien Spiel überläßt als das Grundgesetz. Die Frage, inwieweit der Supreme Court Einfluß auf die Rechtspolitik nimmt, und damit die Frage nach der politischen Funktion des Gerichtes, versucht das hier angezeigte Buch zu beantworten.

Es handelt sich um einen Reader, wie sie an amerikanischen Universitäten üblich sind. Die wesentlichsten Aufsätze und die berühmten politischen Entscheidungen sind enthalten. Das Buch zeichnet sich jedoch durch besonders intelligente Einführungsessays und Bemerkungen zu den Entscheidungen der beiden Autoren aus. Gedacht ist das Buch als Grundlage für einen zweisemestrigen Kurs. Studenten haben nach der Durcharbeitung des Buches aber nicht nur einen Blick für die politische Bedeutung von Supreme-Court-Entscheidungen - was das Hauptanliegen ist - , sondern auch einen Begriff davon, welche Dynamik eine demokratische Verfassung entwickeln kann, wenn sie nicht als bloßes Gesetz, sondern als Grundprogramm für eine gesellschaftliche Entwicklung angesehen wird. Mit diesem Reader ist es den Verfassern gelungen, das eminent Politische der Verfassungsgerichtsbarkeit deutlich zu machen und gleichzeitig vorzuführen, wie stark die verfassungsrechtliche Interpretation von dem gesellschaftlichen Kontext abhängt, aber auch davon, ob es gelingt, Richterpersönlichkeiten $\mathrm{zu}$ finden, die in der Lage sind, die notwendigen gesellschaftlichen Entwicklungen in der Verfassungsrechtsprechung zu fördern, ohne die gemeinsamen Grundüberzeugungen zu gefährden. Das Buch kann allen am amerikanischen System der Verfassungsgerichtsbarkeit Interessierten als Grundlage empfohlen werden.

Ein besonderer Vorzug dieses Buches liegt darin, daß hier die Rechtsprechung und Literatur unter einer speziellen Fragestellung erneut zusammengestellt wird. Eine ideale Ergänzung zu einem allgemeinen Kurs über Verfassungsrechtsprechung, weil gleichzeitig wiederholt und eine besondere Fragestellung verfolgt wird. Es wäre sehr zu begrüßen, wenn Unterrichtsmaterialien solcher Art auch bei uns neben die klassischen Lehrbücher treten würden.

Henning v. Wedel

\title{
Michael KolL
}

Das Entwicklungspotential der Interessenverbände

Materialien des Arnold-Bergstraesser-Instituts Bd. 35

Bertelsmann Universitätsverlag Düsseldorf 1971, 149 Seiten

In der Literatur über Entwicklungsländer sind die Interessenverbände bisher weitgehend unberücksichtigt geblieben. Die vorhandenen Publikationen befassen sich zudem fast ausschließlich mit der Beschreibung bestehender Organisationsformen und dem historischen Werdegang. Das gilt auch für einen Aufsatz des Autors aus dem Jahre 1964 (Soziale Organisation im Wandel - Tendenz zur freiwilligen Vereinigung, in: Materialien Bd. 19 Africana Collecta I, S. 69 ff.), der in vielem als Ausgangspunkt für das vorliegende Buch herangezogen werden kann. 
Das Verdienst von Koll ist es nun, daß er nicht nur die Ergebnisse einer sehr eingehenden empirischen Untersuchung über die Handwerksinnungen in Ibadan vorgelegt hat (S. 31-74), sondern auch der Frage nachgegangen ist, ob und in wieweit in den vorhandenen Organisationen Entwicklungsmöglichkeiten stecken. Die Frage hat eine doppelte Dimension: zum einen die Entwicklung der jeweiligen Innung selbst, zum anderen die gesamtgesellschaftliche. Koll nimmt ohne nähere Diskussion an, daß eine Fortentwicklung der untersuchten Innungen notwendig in einen gesamtgesellschaftlichen Entwicklungserfolg ausmünden müsse und prüft daher lediglich die Entwicklungswürdigkeit und -möglichkeit der Innungen (S 86, 87).

Schon in dem oben genannten Aufsatz hatte Koll die Ansicht vertreten (S. 76/77), $\mathrm{da}$ es bei den wirtschaftlichen Vereinen Afrikas so große Ähnlichkeit mit der Entwicklung in Europa zwischen dem 11. und 16. Jahrhundert gebe, daß man schlechterdings die afrikanische Entwicklung mit europäischen Erfahrungswerken fortschreiben könne, um zu hinreichend genauen Ergebnissen über die Zukunftswirksamkeit der afrikanischen, speziell west-nigerianischen Innungen zu kommen.

Dieser Ansatz nun erscheint aus mehrfacher Sicht bedenklich. Zunächst einmal gibt es in Afrika kein „keimfreies Feld“ mehr, an dem noch auszumachen wäre, ob die behauptete Parallelentwicklung auch ohne die europäischen Vorbilder stattfinden würde, oder ob nicht vielmehr die Beobachtung nur den Grad der Rezeption europäischer Vorstellungen in Afrika widerspiegelt. Zum anderen wäre zu diskutieren, ob denn eine Paralellentwicklung tatsächlich so wünschbar ist, daß der Rolle der Interessenverbände in der BRD, die der Autor mit bewegten Worten schildert (S. $13 \mathrm{ff}$.), eine ähnliche in den Entwicklungsländern an die Seite zu stellen sei. Der Autor weist zwar in Kapitel 4.1 experimentell die Förderungswürdigkeit nach, nicht aber die gesamtgesellschaftliche Entwicklungsnützlichkeit.

Diese Unterlassungen sind nun sicher nicht ein Versäumnis des Autors im Rahmen der hier vorgelegten Vröffentlichung. Der Autor kommt jedoch, bevor er die Arbeit recht kurz auf acht Seiten mit dem möglichen Beitrag der Innungen zur Entwicklung abschließt (S. 86 bis 94), am Ende der empirischen Untersuchung (S. 83) zu dem Ergebnis, daß echte Chancen für Entwicklungsansätze durch die Innungen nur bestehen, wenn die Regierung sich zur Förderung dieses gesellschaftlichen Bereichs verstünde. Das ist aber bisher kaum der Fall. Mir scheint daher, daß es nicht sosehr auf ein Entwicklungspotential der Interessenverbände ankommt, als vielmehr darauf, warum die Regierung dieses Potential vernachlässigt. Dies kann aber wohl nur geklärt werden, wenn man kritisch untersucht, vom Standpunkt des jeweiligen Entwicklungslandes aus, von welcher Qualität gesellschaftlicher und politischer - das Entwicklungspotential der Interessenverbände ist.

Dem Autor ist es zu danken, daß er diese Diskussion angeregt und nützliches Material beigesteuert hat.

Hinrich Schroeder-Hohenwarth 